\title{
Education as absorptive capacity and its role for economic growth
}

\author{
Mourelle, Estefanía ${ }^{\text {; }}$ Márquez-Ramos, Laura ${ }^{\text {b }}$ \\ ${ }^{\mathrm{a}}$ Universidade da Coruña, Spain; ${ }^{\mathrm{b}}$ Institute for International Trade, University of Adelaide, \\ Australia.
}

\begin{abstract}
Might a country's economic growth behave in a different manner depending on the evolution of its absorptive capacity? In this research, education is considered as a channel for economic growth since it is a key element for a country's absorptive capacity. Then, we hypothesize the existence of a threshold for absorptive capacity, so that once it is exceeded, economic growth shows certain (and different) characteristics. Addressing this question requires moving from the traditional linear framework to a nonlinear one and, in this sense, we resort to Smooth Transition specifications. The empirical results point to the existence of nonlinearities in the relationship between absorptive capacity and economic growth. According to the behavior of the country's absorptive capacity, economic growth displays different dynamics; this asymmetric evolution is clearly appreciated in the two dimensions of absorptive capacity taken into account (i.e., secondary and tertiary), although it is more pronounced in case of tertiary education. Our evidence provides important insights for higher education such as the fact that both secondary and tertiary education matter for countries' economic growth and that nonlinearities of this relationship should be taken on board.
\end{abstract}

Keywords: education; absorptive capacity; economic growth; network society; nonlinearities; Smooth Transition models. 


\section{Introduction}

The objective of the present paper is to analyze the relationship between education and economic activity from a new perspective. Specifically, we understand that a country with a population able to exploit new knowledge will perform better. Therefore, education is considered a channel for economic growth since it constitutes an intrinsic mechanism of knowledge absorption ${ }^{1}$ and then, it is a key element for a country's absorptive capacity. Following Cohen and Levinthal (1990), Zahra and George (2002), and Márquez-Ramos and Martínez-Zarzoso (2010), we consider absorptive capacity as the ability to put information from abroad into practice, which plays a key role in economic development. In this vein, we focus on exploitation capabilities and examine their role in economic growth. As a consequence, our interest goes beyond the consideration of years of schooling as a potential determinant of economic growth: we generalize to countries' exploitation capabilities and explore the inherent nonlinearities in the absorptive capacity-economic growth relationship.

This research has a bearing in the literature of education and economic growth, which has analyzed the causal effect of education on countries' economic performance using a variety of measures and quantitative tools for cross-country, time series and panel data. In this brand of the literature, Hanushek and Wößmann (2010) highlighted that economic growth is affected by people's knowledge. Also the importance of nonlinearities has previously been identified, as the work of Krueger and Lindahl (2001) points out.

The present paper brings into question the linearity assumption by using time series techniques for 1971-2013 in Spain, as we believe that the role of absorptive capacity can be that of a force driving a possible nonlinear behavior in the economic growth. Moreover, we incorporate additional variables that are closely related to both absorptive capacity and economic growth, as they also affect this relationship. Focusing on the case of Spain is of great interest, mainly because Spain is a country with a solid trajectory in its education system and it is also a developed economy that is representative of a large number of countries. In this case study, we are able to provide evidence of the nonlinear relationship between absorptive capacity and economic growth at country-level. This analysis will also help us to shed light on the question posed by the existing literature about the positive, although still without consensus, impact of education on growth.

The paper is organized as follows. Section 2 presents the methodology employed. Section 3 explains the empirical results obtained. Section 4 concludes.

\footnotetext{
${ }^{1}$ See, for example, the illustration of the role of education in the aerospace sector for establishing sufficient levels of absorptive capacity in Asia (van der Heiden et al., 2015).
} 


\section{Methodology}

One common assumption when analyzing the relationship between two variables is that of linearity. Actually, the majority of empirical papers centered on the education-economic growth link are grounded on the linear consideration. Nevertheless, this assumption is too strong if what we aim to analyze is the relationship between absorptive capacity and economic growth, as this would mean that the parameters in the relationship do not change over time. This is, however, unrealistic as it would reflect that learning and innovation processes are linear (Márquez-Ramos and Mourelle, 2016). By using Smooth Transition Regression (STR) models, we allow for the parameters to change depending on a shock, which is defined on an ad hoc basis. This framework provides us with more flexibility when studying the dynamics of economic growth and other variables related to absorptive capacity. The reason for selecting this type of models is in line with the idea of a potential threshold that determines the behavior of the economic growth according to some variable.

Smooth Transition (ST) models belong to the family of state-dependent models where the data-generating process is linear but switches between a certain number of regimes according to some rule. STs have been popular in economic time series, proving good performance in capturing cyclical behavior in macroeconomic variables. For further details on these models, see Teräsvirta $(1994,1998)$ and van Dijk et al. (2002).

We resort to the most general ST model, the STR, as it permits the incorporation of exogenous variables in addition to the endogenous structure. Let $\mathrm{y}_{\mathrm{t}}$ be a stationary, ergodic process and, without loss of generality, one exogenous variable $\mathrm{x}_{\mathrm{t}}$. The model is given by:

$y_{t}=w_{t}^{\prime} \pi+\left(w_{t}^{\prime} \theta\right) F\left(s_{t} ; \gamma, c\right)+u_{t}$,

with $\quad w_{t}=\left(1, y_{t-1}, \ldots, y_{t-p 1} ; x_{t}, x_{t-1}, \ldots, x_{t-p 2}\right)^{\prime} \quad$ a $\quad$ vector $\quad$ of regressors; $\pi=\left(\pi_{0}, \pi_{1}, \ldots, \pi_{p}\right)^{\prime}$ and $\theta=\left(\theta_{0}, \theta_{1}, \ldots, \theta_{p}\right)^{\prime}$ parameter vectors $\left(\mathrm{p}=\mathrm{p}_{1}+\mathrm{p}_{2}+1\right)$; and $\mathrm{u}_{\mathrm{t}}$ an error process. $\mathrm{F}($.) is a transition function customarily bounded between 0 and 1 , so that STR coefficients vary between $\pi_{\mathrm{j}}$ and $\pi_{\mathrm{j}}+\theta_{\mathrm{j}}(\mathrm{j}=0, \ldots, \mathrm{p})$, respectively. The regime at each $\mathrm{t}$ is determined by the transition variable, $\mathrm{s}_{\mathrm{t}}$, and the associated value $\mathrm{F}\left(\mathrm{s}_{\mathrm{t}}\right)$; the transition variable can be a lagged endogenous variable, an exogenous variable or another variable. The slope parameter $\gamma$ defines the smoothness of the transition: the higher it is, the more rapid the change; the location parameter $\mathrm{c}$ indicates the threshold between the two regimes.

Two formulations are mainly used for F: logistic and exponential. In the logistic model the extreme regimes are associated with $\mathrm{s}_{\mathrm{t}}$ values far above or below $\mathrm{c}$, where dynamics may be different; otherwise, in the exponential case the extreme regimes are related to low and high absolute values of $\mathrm{s}_{\mathrm{t}}$, with rather similar dynamics, which can be different in the transition 
period. Conceptually speaking, the logistic transition appears to be the most suitable for describing our relationship, as there is no reason to assume a similar effect for a positive and a negative variation in the absorptive capacity of a country on its economic activity.

The STR model is estimated by nonlinear least squares. Following the related literature, we carry out an extensive search of STRs by defining a grid for $(\gamma, c)$, trying for different values of $\gamma$ and using the mean of the transition variable for $\mathrm{c}$. Where parameter convergence is reached, models are subject to further refinement; we evaluate cross-parameter restrictions to increase efficiency and drop nonsignificant coefficients to conserve degrees of freedom.

In the ST framework, special attention is given to the validation stage, as it unveils any possible inadequacy of the model. Most tests commonly applied to dynamic models are also valid for STs. Besides, there are evaluation tests especially derived for STs: the test of residual serial independence; the test of no remaining nonlinearity in the residuals; and the test of parameter constancy. In this paper, we use the adjusted $\mathrm{R}^{2}$ and the variance ratio of the residuals from the nonlinear model and the linear regression estimated by Ordinary Least Squares (OLS), as it provides relevant information on the explanatory power of both models. In addition, we use the test of no ARCH structure and the specific STs tests.

Finally, in order to offer a better characterization of the relationship under study, we also develop a structural analysis of the STR model through the definition and understanding of the theoretical paths economic growth would follow in response to changes in education.

\section{Empirical results}

\subsection{Data and preliminary analysis}

As mentioned above, the data used for this empirical application is centered on the Spanish case. Real and seasonally adjusted Gross Domestic Product (GDP) is obtained from the International Monetary Fund's IFS database; GDP is traditionally considered for measuring economic activity. We measure absorptive capacity by using gross enrollment ratios for secondary and tertiary education (available from the World Bank); we denominate dimension 1 to our measure for secondary education and dimension 2 to tertiary education. The proxies for labor force are defined by calculating the proportion of active population with secondary (tertiary) education over total active population (data obtained from The Valencian Institute of Economic Research, IVIE); in the context of our research, these variables proxy for the prior knowledge base in Spain. For the physical capital, we use real Gross Fixed Capital Formation (IVIE database); we include investment as it exerts an indirect effect on education. Finally, the government expenditure on education (\% of GDP, World Bank) serves as a control variable that allows to isolate the relationship between absorptive capacity and economic growth. The sample goes from 1971 to 2013 and we use annual data. All variables are used in logarithms. Figure 1 depicts the variables over time. 


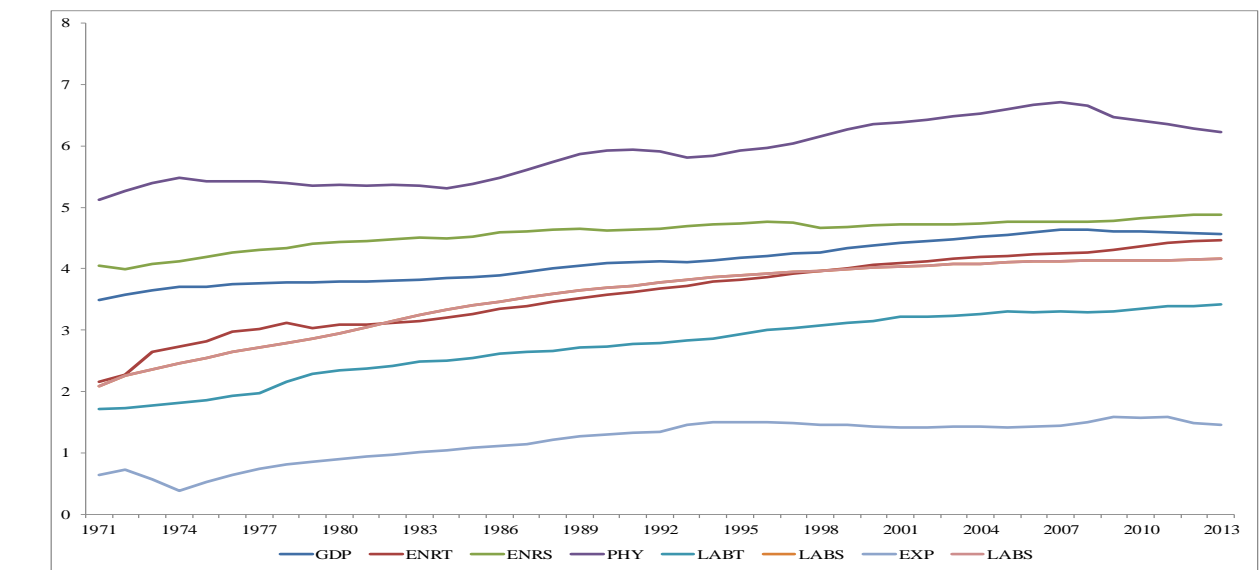

Figure 1. Evolution of the variables over time, in logs. Source: Own elaboration. Notes. ENRT: enrollment ratio tertiary education (ENRS for secondary education); PHY: physical capital; LABT: labor force tertiary education (LABS for secondary education); EXP: government expenditure on education.

Before focusing on the relationship between economic growth and absorptive capacity, a statistical processing of the information is required. In particular, all the variables involved in the study must be stationary, a property that is evaluated by means of unit root tests. In this sense we have applied the Ng and Perron (2001) unit root tests. Results are not shown for space reasons ${ }^{2}$. All the variables considered in the study are unit root processes. These results point out the need for applying regular differences in all our variables.

\subsection{Estimated models: Discussion}

Three aspects must be remarked. First, as specific data regarding enrollment and active population ratios for secondary and tertiary education is available, the estimation process has two branches so as to distinguish the two dimensions of absorptive capacity. Second, four explanatory variables are initially included in the analysis: education, physical capital, labor force and expenditure on education. Third, we consider up to two lags of the variables in the regressions to account for the effect of their most recent history on economic growth.

The starting point of the modelling procedure consists of finding out the linear model that would describe the behavior of our series. OLS estimation is carried out; all parameters are introduced initially, but then those nonsignificant at a 0.05 level are successively excluded (results not reported). The next stage is the estimation of the nonlinear models. We achieve valid STR specifications for the secondary and tertiary education. As our main purpose is to study the impact of absorptive capacity on economic growth, the decision on the transition variable is clear: the enrollment ratio. Then, this variable plays a double role in explaining the economic growth dynamics. On the one hand, it represents the source of nonlinearities

\footnotetext{
${ }^{2}$ Along the paper, all the results not shown for space reasons are available from the authors upon request.
} 
in the evolution of economic growth; on the other hand, it is also a determining factor of GDP growth dynamics. Table 1 presents the estimated models, along with several diagnostic statistics and evaluation tests.

Table 1. Estimated STR models

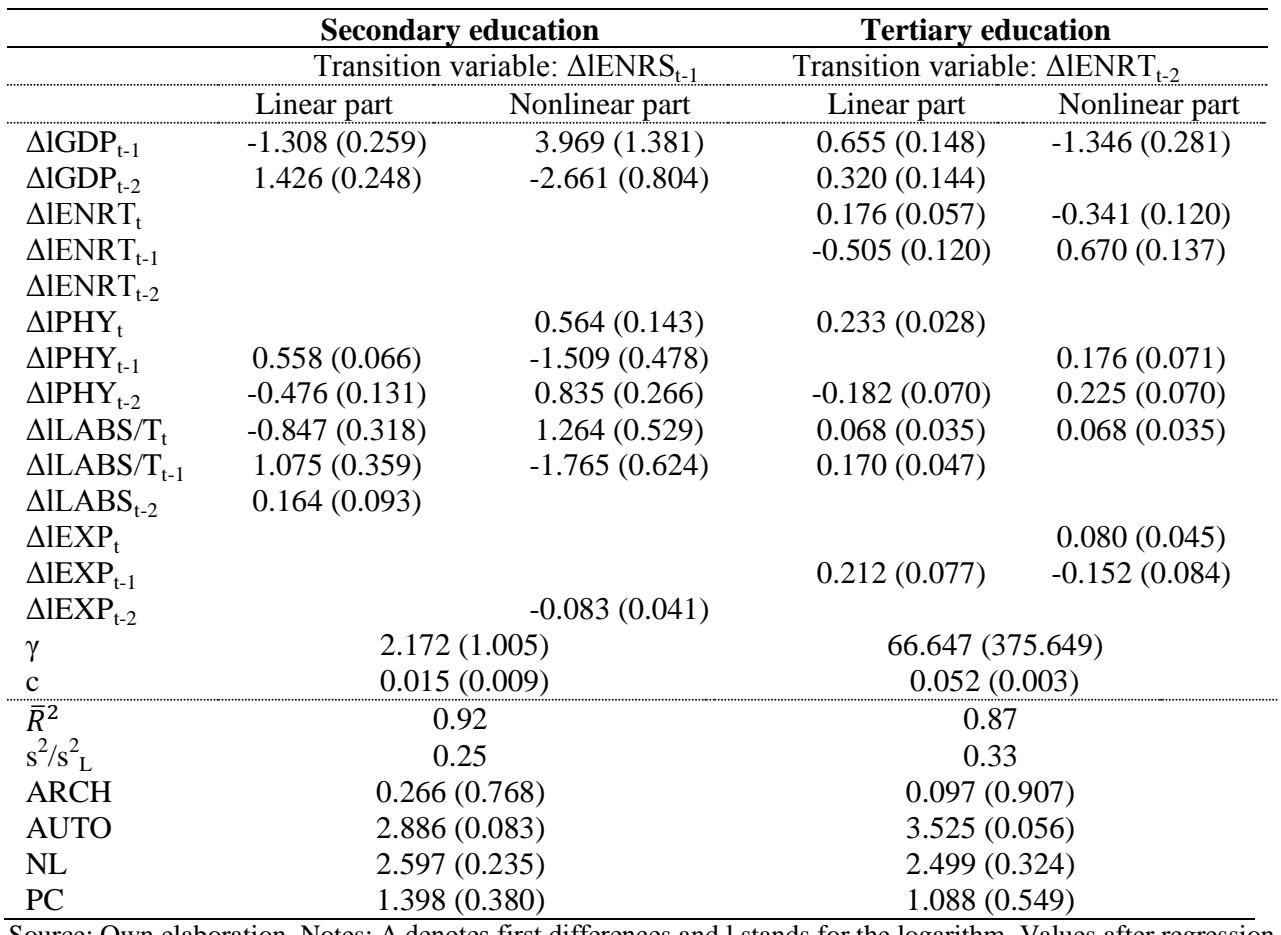

Source: Own elaboration. Notes: $\Delta$ denotes first differences and 1 stands for the logarithm. Values after regression coefficients are SEs of the estimates; $\bar{R}^{2}$ : adjusted determination coefficient; $\mathrm{s}^{2} / \mathrm{s}_{\mathrm{L}}^{2}$ : variance ratio of the residuals from the STR model and the linear regression; ARCH: statistic of no ARCH based on 2 lags; AUTO: test for residual autocorrelation of order 2; NL: test for no remaining nonlinearity; PC: general parameter constancy test. Numbers in parentheses after evaluation statistics are p-values.

Going into detail on dimension 1 of exploitation capabilities, we appreciate notably significant coefficients in the estimated model that highlight the dependence of economic growth on its recent history, as well as on physical capital, labor force and expenditure on education. As it is observed, the first dimension of absorptive capacity is not a determining factor for economic activity. Figure 2 depicts the estimated transition functions. 

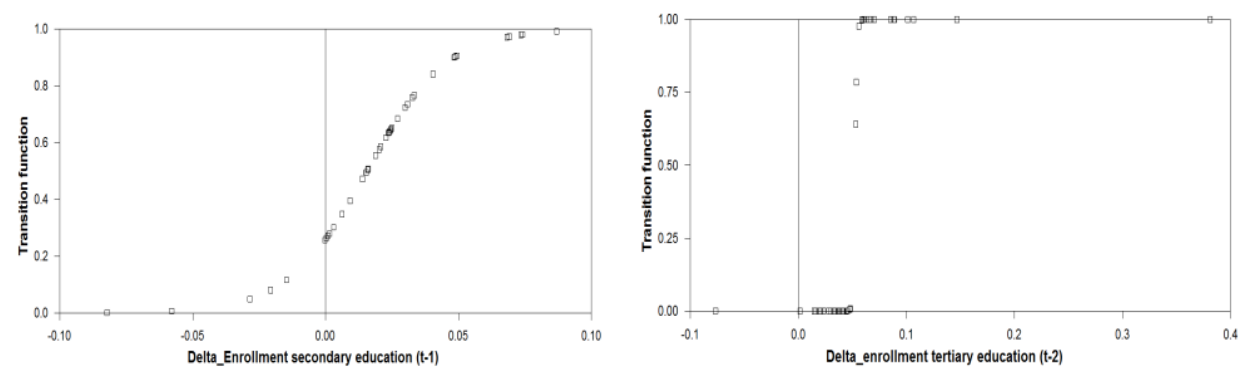

Figure 2. Estimated transition functions. Source: Own elaboration.

The transition between regimes is smooth, according to $\gamma$, and the delay is one year. We can define a lower extreme regime, covering from negative growth to $1.5 \%$ variation in the enrollment ratio, and an upper extreme regime, for rates greater than $1.5 \%$. The estimated threshold is slightly below the enrollment ratio mean (2\%). The model presents no evidence of misspecification; there are no signs of $\mathrm{ARCH}$ structure and the validation tests do not detect serial dependence in the estimated residuals, there is no need for a second transition function and parameter constancy is demonstrated. A fact to emphasize is the high explanatory power of this model compared to the linear one: according to the variance ratio, the STR model explains $75 \%$ of the residual variance of the linear regression.

Turning now to the second dimension of exploitation capabilities, the estimated parameters are again highly significant, indicating that recent history of economic growth has effects on its current state, as well as enrollment ratio, physical capital, labor force and expenditure on education. In contrast to the previous dimension, the enrollment ratio in tertiary level is a significant determinant for the economic growth dynamics in Spain. This result indicates that higher education is a key factor for economic development and, in terms of absorptive capacity, we interpret that it is very important for economic growth that people in a country are "creators" and not only "users" of those tools required for processing innovation and creation. We consider that a higher number of "creators" in a country increases the country's potential to put new information and knowledge into practice by developing new products and processes that conduct to economic growth.

Another difference with secondary education are the features of the transition from one regime to the other: now it is quite abrupt and the delay is two years instead of one. In fact, these extremely rapid regime changes suggest the need for threshold specifications, strengthening the importance of using STR models. Therefore, economic growth evolves more rapidly from one extreme regime to the other when considering the second dimension of absorptive capacity; that is, economic growth reacts in a more immediate way to shocks in tertiary education. A possible cause can be found in the high weight traditionally given to pursuing an university degree in Spain; a historical and sociological background points to a more rooted character of higher education in Spain than in other similar countries. 
Two regimes arise from this model: a lower extreme regime, ranging from negative growth to $5.2 \%$ variation in the enrollment ratio, and an upper extreme regime, for variations greater than $5.2 \%$. The threshold is remarkably close to the mean of the transition variable (5.7\%). There are no indications of misspecification, so one may conclude that this STR model is adequate. Once again, this model shows greater explanatory power than the linear formulation: the STR model explains $67 \%$ of the residual variance of the linear regression.

\subsection{Short-run dynamics analysis}

This study consists of analyzing the path that economic growth - whose behavior is described by a nonlinear model - would keep after the effect of changes in absorptive capacity. As a result, the immediate effect of these changes (in sign and quantity) and how the variable returns to an equilibrium path can be appreciated. Two considerations must be taken into account beforehand. First, the starting value for the variation of the proxies used for measuring absorptive capacity (i.e., enrollment ratios) corresponds to its sample mean. Second, the values of the other regressors remain constant at a certain level (its sample mean) when examining the effect of a change in the enrollment ratio on economic growth.

We simulate the evolution of economic growth according to its behavior defined by the STR model. Regarding the first dimension of absorptive capacity, the point of departure is a stationary equilibrium state characterized by a $1 \%$ enrollment ratio rate of growth (the mean rate for this variable is below $2 \%$ in our sample). In the first exercise, the enrollment ratio passes from growing at a $1 \%$ to a $1.5 \%$. This evolution causes an increase in economic growth in the short run; the impact is quite outstanding at the beginning and moderate (even negative) afterwards. The return to an equilibrium growth is achieved several years later.

A further distinctive feature of nonlinear models is the lack of symmetry in the responses of the variables aftershocks (the linear context implies symmetry). Along these lines, we carry out a second exercise, where the enrollment ratio steadily rises at a $1 \%$ until it starts to rise at a $0.5 \%$. This slower pace involves a severe fall in economic growth; the impact is quite irregular but always means negative values, and the path towards the stationary location takes longer than in the previous exercise. We clearly observe the absence of symmetry when considering two comparable situations (plus or minus five percentage points).

Regarding the second dimension of absorptive capacity, the starting point is a stationary equilibrium state characterized by a $3 \%$ enrollment ratio growth rate (the mean rate for this variable is below $6 \%$ in our sample). In the new exercise, the enrollment ratio starts to grow at a higher rate $(5 \%)$ than the initial one $(3 \%)$. Following the theoretical path, the economic growth reacts with a moderate increase in its growth level; after several years, the variable begins to fall to the long-run location. In the last exercise, the enrollment ratio experiences a decrease in its growth rate (from a $3 \%$ to a $1 \%$ ). The evolution of the economic growth 
turns unfavorable after this shock; economic activity grows at almost zero rates during the first years but then faces with negative rates. We appreciate again the lack of symmetry.

In short, the simulation study appears to point to a direct impact of absorptive capacity in the short run (with the two dimensions) on economic growth. As a remarkable fact, the effects become more severe when the enrollment ratio (and hence absorptive capacity) decreases its growth rate; in this case, a decrease in absorptive capacity impacts economic growth generating a larger negative effect when there is a decrease in the number of "users" (i.e. secondary education) than in the number of "creators" (i.e. tertiary education) of those tools required in the society. An explanation may rest on the fact that secondary education, apart from an objective itself, is also the previous requirement for higher education studies; in other words, a certain proportion of "users" will be "creators" in the future.

An important conclusion we can draw from the analysis is that in order to economically succeed, it seems necessary that countries maintain and foster their level of absorptive capacity, not only by maintaining (or increasing) their achieved number of "users" of those tools required, but also by increasing the number of "creators". Therefore, under the contemporaneous societal changes that we are experiencing worldwide, going from an industrial society, to a knowledge society and, finally, to a network, the future role of universities and other higher education organizations should be a priority for the development of countries.

\section{Conclusions}

This paper analyzes the nonlinear relationship between absorptive capacity and economic growth at country-level. To do so, it focuses on secondary and tertiary enrollment in Spain. The empirical framework is that of time series (from 1971 to 2013) and, more specifically, we estimate Smooth Transition Regressions, which are able to explain our relationship of interest in a satisfactory manner. The asymmetric evolution of economic growth is clearly observable in the two dimensions of absorptive capacity taken on board (secondary and tertiary), although it is more pronounced in the case of higher education. In sum, this research proves the crucial relevance of education, and of higher education in particular. Our main policy implication is that governments must care for their educational system, not only for its intrinsic importance, but also for its effects on a country's economic growth. 


\section{References}

Cohen, W.M., \& Levinthal, D.A. (1990). Absorptive capacity: A new perspective on learning and innovation. Administrative Science Quarterly, 35, 128-152.

Hanushek, E.A., \& Wößmann, L. (2010). Education and economic growth. In P. Peterson, E. Baker \& B. McGaw (Eds.), International Encyclopedia of Education. Volume 2 (pp. 245-252). Oxford: Elsevier.

Krueger, A.B., \& Lindahl, M. (2001). Education for growth: Why and for whom? Journal of Economic Literature, 39, 1101-1136.

Márquez-Ramos, L., \& Martínez-Zarzoso, I. (2010). The effect of technological innovation on international trade. Economics: The Open-Access, Open-Assessment E-Journal, 4 (2010-11), 1-37.

Márquez-Ramos, L., \& Mourelle, E. (2016). Selecting a suitable approach to analyze the future of higher education. Procedia - Social and Behavioral Sciences, 228, 86-91.

Teräsvirta, T. (1994). Specification, estimation, and evaluation of smooth transition autoregressive models. Journal of the American Statistical Association, 89(425), 208218.

-. (1998). Modeling economic relationships with smooth transition regressions. In A. Ullah \& D.E.A. Giles (Eds.), Handbook of Applied Economic Statistics (pp. 507-552), New York: Marcel Dekker.

van Dijk, D., Teräsvirta, T., \& Franses, P.H. (2002). Smooth transition autoregressive models-A survey of recent developments. Econometric Reviews, 21(1), 1-47.

van der Heiden, P., Pohl, C., Mansor, S.B., \& van Genderen, J. (2015). The role of education and training in absorptive capacity of international technology transfer in the aerospace sector. Progress in Aerospace Sciences, 76, $42-54$.

Zahra, S. A., \& George, G. (2002). Absorptive capacity: A review, reconceptualization, and extension. Academy of Management Review, 27(2), 185-203. 